\title{
Near Optimal Common Detection Techniques for Shaped Offset QPSK and Feher's QPSK
}

\author{
Tom Nelson \\ Michael D. Rice \\ mdr@byu.edu \\ Erik Perrins
}

Follow this and additional works at: https://scholarsarchive.byu.edu/facpub

Part of the Electrical and Computer Engineering Commons

\section{Original Publication Citation}

Tom Nelson, Erik Perrins, and Michael Rice. "Near Optimal Common Detection Techniques for Shaped Offset QPSK and Feher's QPSK," IEEE Transactions on Communications, Vol. 56, No. 5, May 2008. 724-735. DOI: 10.1109/TCOMM.2008.060155

\section{BYU ScholarsArchive Citation}

Nelson, Tom; Rice, Michael D.; and Perrins, Erik, "Near Optimal Common Detection Techniques for Shaped Offset QPSK and Feher's QPSK" (2008). Faculty Publications. 1226.

https://scholarsarchive.byu.edu/facpub/1226

This Peer-Reviewed Article is brought to you for free and open access by BYU ScholarsArchive. It has been accepted for inclusion in Faculty Publications by an authorized administrator of BYU ScholarsArchive. For more information, please contact ellen_amatangelo@byu.edu. 


\title{
Near Optimal Common Detection Techniques for Shaped Offset QPSK and Feher's QPSK
}

\author{
Tom Nelson*, Erik Perrins ${ }^{\dagger}$, and Michael Rice* \\ *Department of Electrical \& Computer Engineering, Brigham Young University, \\ Provo, UT 84602 \\ ${ }^{\dagger}$ Department of Electrical Engineering \& Computer Science, University of Kansas, \\ Lawrence, KS 66045
}

\begin{abstract}
This paper compares three candidate detection techniques for shaped offset QPSK (SOQPSK-TG) and Feher's QPSK (FQPSK-JR), modulations which are embodied as fully interoperable modulations in the Interrange Instrumentation Group standard IRIG-106. The techniques are based on a common trellis-coded modulation representation and a common continuous phase modulation (CPM) representation for these two modulations. In addition the pulse amplitude modulation (PAM) decomposition of the common CPM representation is developed. All three techniques achieve near-optimal bit error rate performance. The PAM-based detector offers the best performance-complexity trade-off.
\end{abstract}

\section{INTRODUCTION}

Power and bandwidth constraints present challenges to modulation design. The constant envelope constraint is also imposed when operation through a fully saturated non-linear RF power amplifier is required. Examples include commercial and military satellite communication links, digital mobile telephony (i.e., Gaussian minimum shift keying (GMSK) for the Global System for Mobile communications (GSM) [1]), and aeronautical telemetry [2].

Aeronautical telemetry is an interesting case study since the solution to this problem resulted in the adoption of two interoperable waveforms known as Feher-patented QPSK (FQPSK) and shaped offset QPSK (SOQPSK). From the 1970s, pulse code modulation/frequency modulation (PCM/FM) has been the dominant modulation used for test and evaluation on government test ranges in the USA, Europe, and Asia. (PCM/FM is binary CPM with a digital modulation index $h=0.7$ and a low-pass filtered 1-REC frequency pulse.) In the USA, the main spectral allocations for aeronautical telemetry are L-band (1435 - $1535 \mathrm{MHz})$, lower S-band (2200 - $2290 \mathrm{MHz})$, and upper S-band (2310 - $2390 \mathrm{MHz})$. Increasing data rate requirements along with an ever increasing number of test flights put tremendous pressure on these spectral allocations in the 1980s and 1990s. The situation was further exacerbated in 1997 when the lower portion of upper S-band from 2310 to $2360 \mathrm{MHz}$ was reallocated in two separate auctions ${ }^{1}$.

\footnotetext{
${ }^{1} 2320$ - $2345 \mathrm{MHz}$ was reallocated for digital audio radio in one auction while $2305-2320 \mathrm{MHz}$ and $2345-2360 \mathrm{MHz}$ were allocated to wireless communications services in the other auction.
} 
In response to this situation, the Telemetry Group of the Range Commander's Council adopted a more bandwidth efficient modulation as part of its IRIG-106 standard [3] in 2000. This modulation, known as FQPSK-B, was a proprietary version of Feher-patented QPSK (FQPSK) described in [4]. Efforts to reduce some aspects of the implementation complexity resulted in a non-proprietary version, known as FQPSK-JR [5] which was adopted as part of IRIG-106 in 2004. Also in 2004, a version of SOQPSK, known as SOQPSK-TG [6], was adopted as a license-free, fully interoperable alternative in the IRIG-106 standard.

These modulations are described in more detail in Section II. Briefly, FQPSK (and its variants) is a linear modulation whose inphase and quadrature components are drawn from a set of waveforms in a constrained way. The set of waveforms, called "wavelets" in the original patents [7], are defined to produce a quasiconstant envelope modulated carrier. (The quadrature waveforms can be defined as delayed versions of the inphase waveforms thereby giving the modulation the look and feel of an offset modulation.) Simon's pioneering analysis of this waveform revealed that the waveform selection constraints can be formulated as a trellis code and termed this, and the general class of modulations, cross-correlated trellis-coded quadrature modulation or XTCQM. SOQPSK-TG is defined as a constrained ternary partial response CPM with modulation index $h=1 / 2$ and was derived from the version of SOQPSK defined in the military UHF SATCOM standard MIL-STD 188-181 [8].

In most situations, a trellis-coded linear modulation and a CPM are not adopted as equivalent transmission techniques in a standard. In this case, the difficulties of adopting a standard with proprietary components and the challenges of licensing patented technology proved the dominant factors in arriving at this odd situation. FQPSK-JR and SOQPSK-TG can coexist in a standard because both have essentially the same bandwidth and the same bit error rate performance when detected using a simple integrate-anddump offset QPSK detector. In the absence of errors, the simple symbol-by-symbol detector produces exactly the same sequence when the transmitter uses either FQPSK-JR or SOQPSK-TG. It is in this sense that the modulations are considered fully interoperable.

The simple symbol-by-symbol detector has two attractive features: 1) low complexity, and 2) it does not have to "know" which modulation is used by the transmitter. These features are achieved at the expense of detection efficiency: the bit error rate performance of this simple detector is about $2 \mathrm{~dB}$ worse than what could be achieved with maximum likelihood detection. Since SOQPSK-TG is a CPM and FQPSK-JR is an XTCQM, it is natural to assume that the optimal detector must be equipped with two different 
detection algorithms and endowed with the knowledge of which modulation is used by the transmitter. In this paper we show that a single detection algorithm can be used for both modulations and that this algorithm does not have to "know" which modulation is used. We refer to such a detector as a common detector and show that its bit error rate performance for both SOQPSK-TG and FQPSK-JR is within 0.1 $\mathrm{dB}$ of the maximum likelihood performance for each.

While considering candidates for the common detector we will compare each detector's bit error rate performance for the two modulations in question to the optimum performance for each of those modulations. SOQPSK-TG and FQPSK-JR have similar distance properties and hence their optimum detectors have similar probabilities of bit error. To facilitate these comparisons for SOQPSK-TG, we define the performance metric

$$
\Delta_{\mathrm{S}}=\left(\frac{E_{b}}{N_{0}}\right)_{\mathrm{C}, \mathrm{S}}-10.21 \mathrm{~dB}
$$

where $\left(E_{b} / N_{0}\right)_{\mathrm{C}, \mathrm{S}}$ is the SNR the common detector requires to achieve $P_{b}=10^{-5}$ when SOQPSK-TG is transmitted and $10.21 \mathrm{~dB}$ is the SNR required for the optimum detector to achive the same probability of bit error. For FQPSK-JR, we define,

$$
\Delta_{\mathrm{F}}=\left(\frac{E_{b}}{N_{0}}\right)_{\mathrm{C}, \mathrm{F}}-10.32 \mathrm{~dB}
$$

where $\left(E_{b} / N_{0}\right)_{\mathrm{C}, \mathrm{F}}$ is the SNR required for the common detector to achive $P_{b}=10^{-5}$ when FQPSK-JR is transmitted and $10.32 \mathrm{~dB}$ is the SNR required for the maximum likelihood FQPSK-JR detector to achieve $P_{b}=10^{-5} . \Delta_{\mathrm{S}}$ and $\Delta_{\mathrm{F}}$ quantify the detection efficiency loss for the candidate common detectors and will be used as figures of merit in this paper.

The paper proceeds as follows: SOQPSK-TG is shown to have an equivalent XTCQM representation in Section III. The corresponding maximum likelihood detector, which follows naturally from this representation, is modified to form an XTCQM detector that is fully compatible with both SOQPSK-TG and FQPSK-JR. The common XTCQM detector has $\Delta_{\mathrm{S}}=0.14 \mathrm{~dB}$ and $\Delta_{\mathrm{F}}=0.08 \mathrm{~dB}$. In Section IVA the equivalent CPM representation for FQPSK-JR derived in Appendix B is simplified and used to produce a common CPM detector. For the common CPM detector $\Delta_{\mathrm{S}}=0.21 \mathrm{~dB}$ and $\Delta_{\mathrm{F}}=0.01 \mathrm{~dB}$. In Section IV-B the PAM decompositions of the CPM representations of these two modulations are used to obtain a common PAM detector. With the common PAM detector $\Delta_{\mathrm{S}}=0.11 \mathrm{~dB}$ and $\Delta_{\mathrm{F}}=0.09 \mathrm{~dB}$. Conclusions and a comparison of the bit error rate performance and complexity of these three common detectors are presented in Section V. 


\section{INTEROPERABLE MODULATIONS}

\section{A. SOQPSK-TG}

SOQPSK-TG is defined as a CPM of the form

$$
s_{\mathrm{S}}(t, \boldsymbol{\alpha})=\sqrt{\frac{2 E_{b}}{T_{b}}} \exp \left[j\left(\phi(t, \boldsymbol{\alpha})+\phi_{0}\right)\right] .
$$

The phase is

$$
\begin{aligned}
\phi(t, \boldsymbol{\alpha}) & =2 \pi h \int_{-\infty}^{t} \sum_{n=-\infty}^{\infty} \alpha_{n} g_{\mathrm{S}}\left(\tau-n T_{b}\right) d \tau \\
& =2 \pi h \sum_{n=-\infty}^{\infty} \alpha_{n} q_{\mathrm{S}}\left(t-n T_{b}\right) .
\end{aligned}
$$

where $g_{\mathrm{S}}(t)$ is the frequency pulse; $q_{\mathrm{S}}(t)=\int_{-\infty}^{t} g_{\mathrm{S}}(\tau) d \tau$ is the phase pulse; $\phi_{0}$ is an arbitrary phase which, without loss of generality, can be set to $0 ; T_{b}$ is the bit interval (or reciprocal of the bit rate); $h=1 / 2$ is the modulation index; and $\alpha_{n} \in\{-1,0,1\}$ are the ternary symbols which are related to the binary input symbols $a_{n} \in\{-1,1\}$ by [9]

$$
\alpha_{n}=(-1)^{n+1} \frac{a_{n-1}\left(a_{n}-a_{n-2}\right)}{2} .
$$

The frequency pulse for SOQPSK-TG is a spectral raised cosine windowed by a temporal raised cosine [6]:

$$
g_{\mathrm{S}}(t)=C \frac{\cos \left(\frac{\pi \rho B t}{2 T_{b}}\right)}{1-4\left(\frac{\rho B t}{2 T_{b}}\right)^{2}} \times \frac{\sin \left(\frac{\pi B t}{2 T_{b}}\right)}{\left(\frac{\pi B t}{2 T_{b}}\right)} \times w(t)
$$

for

$$
w(t)= \begin{cases}1 & 0 \leq\left|\frac{t}{2 T_{b}}\right|<T_{1} \\ \frac{1}{2}+\frac{1}{2} \cos \left(\frac{\pi}{T_{2}}\left(\frac{t}{2 T_{b}}-T_{1}\right)\right) & T_{1} \leq\left|\frac{t}{2 T_{b}}\right| \leq T_{1}+T_{2} . \\ 0 & T_{1}+T_{2}<\left|\frac{t}{2 T_{b}}\right|\end{cases}
$$

For SOQPSK-TG, the parameters are $\operatorname{ar}^{2} \rho=0.7, B=1.25, T_{1}=1.5$, and $T_{2}=0.5$. The constant $C$ is chosen to make $q_{\mathrm{S}}(t)=1 / 2$ for $t \geq 2\left(T_{1}+T_{2}\right) T_{b}$. The frequency pulse and corresponding phase pulse for this case are shown in Figure 1. Observe that these values of $\rho, B, T_{1}$ and $T_{2}$ make SOQPSK-TG a partial response CPM spanning $L=8$ bit intervals.

\footnotetext{
${ }^{2}$ In the original publication [6], two versions of SOQPSK were described: SOQPSK-A defined by $\rho=1, B=1.35, T_{1}=1.4$, and $T_{2}=0.6$ and SOQPSK-B defined by $\rho=0.5, B=1.45, T_{1}=2.8$, and $T_{2}=1.2$. SOQPSK-A has a slightly narrower bandwidth (measured at the $-60 \mathrm{~dB}$ level) and slightly worse detection efficiency than SOQPSK-B. The Telemetry Group of the Range Commanders Council adopted the compromise waveform, designated SOQPSK-TG in 2004.
} 
An analysis of maximum likelihood detection of SOQPSK was performed by Geoghegan [10], [11], [12] following the standard union bound technique based on pairwise error probabilities [13]. The binaryto-ternary mapping (5) contributes an extra step to the analysis. Let

$$
\mathbf{a}=\ldots a_{k-3}, a_{k-2}, a_{k-1}, a_{k}, a_{k+1}, a_{k+2}, a_{k+3}, \ldots
$$

represent a generic binary symbol sequence with $a_{k} \in\{-1,+1\}$. The minimum distance error event occurs between the waveforms corresponding to two binary symbol sequences whose difference satisfies

$$
\mathbf{a}_{1}-\mathbf{a}_{2}= \pm[\ldots, 0,0,0,2,0,0,0, \ldots]
$$

where the difference (or erroneous symbol) occurs at index $k$. There are 64 such sequence pairs. As it turns out, there are two ways a pair of binary sequences can produce (9). Sequence pairs for which $a_{k-1}=a_{k+1}$ are characterized by waveforms separated by a normalized squared Euclidean distance of 1.60. There are 32 such sequences. Sequence pairs for which $a_{k-1}=-a_{k+1}$ are characterized by waveforms separated by a normalized squared Euclidean distance of 2.58. There are 32 such sequences. Since these error events produce one bit error, the probability of error is well approximated by

$$
P_{b} \approx 1 \times \frac{32}{64} Q\left(\sqrt{1.60 \frac{E_{b}}{N_{0}}}\right)+1 \times \frac{32}{64} Q\left(\sqrt{2.58 \frac{E_{b}}{N_{0}}}\right) .
$$

(Why the coefficients are written this way will become evident in Section IV.) Using this expression, $P_{b}=10^{-5}$ is achieved at $E_{b} / N_{0}=10.21 \mathrm{~dB}$.

\section{B. FQPSK-JR}

FQPSK-JR is defined as an offset QPSK modulation of the form

$$
s_{\mathrm{F}}(t)=\sum_{n} s_{I, m}\left(t-n T_{s}\right)+j s_{Q, m}\left(t-n T_{s}-T_{s} / 2\right) .
$$

with data dependent pulses $s_{I, m}(t)$ and $s_{Q, m}(t)$ each drawn in a constrained way from a set of 16 waveforms [5]. The waveform index $m$ is determined by the modulating data bits as explained in [14]. The 16 pulses have a duration of $2 T_{b}=T_{s}$ and are listed in [5] and [14]. Simon showed that the original version of FQPSK has an XTCQM interpretation from which the optimum maximum likelihood detector followed [14]. This representation consists of 16 waveforms for the inphase component and 16 waveforms for the quadrature component for a total of 32 possible complex-valued waveforms when the constraints on possible combinations are taken into account. The XTCQM representation of FQPSK-JR is the same as that for the original FQPSK except that three of the waveforms are modified. Consequently the optimum detector for FQPSK-JR has the same form as that described by Simon [14] for FQPSK. 
For the purposes of comparison with the XTCQM representation of SOQPSK-TG, it is advantageous to re-express FQPSK-JR in the form

$$
s_{\mathrm{F}}(t)=\sum_{k} I_{\mathrm{F}}\left(t-k T_{s} ; a_{2 k}, \ldots, a_{2 k-4}\right)+j Q_{\mathrm{F}}\left(t-k T_{s} ; a_{2 k}, \ldots, a_{2 k-4}\right) .
$$

Five information bits are used to select an in-phase waveform $I_{\mathrm{F}}(t ; \cdot)$ and a quadrature waveform $Q_{\mathrm{F}}(t ; \cdot)$ which are transmitted during an interval of $2 T_{b}$ seconds. The next waveform is determined by clocking in two new bits (and discarding the two oldest bits) to form a new group of 5 bits that select the waveform. This slightly different, but equivalent, point of view represents the memory in the modulated carrier using a sliding window that is five bits wide and strides through the input data 2 bits at a time. Note that $I_{\mathrm{F}}(t ; \cdot)$ is drawn from a set of 16 waveforms (the $s_{I, m}(t)$ of (11) which are uniquely specified using 4 bits) but that the addressing uses 5 bits. This is explained as follows: When FQPSK is expressed in the form (12), the list of 32 waveforms for $I_{\mathrm{F}}(t ; \cdot)$ consists of the 16 waveforms $s_{I, m}(t)$ of $(11)$ each repeated twice. The same applies to $Q_{\mathrm{F}}(t ; \cdot)$ together with $s_{Q, m}(t)$ of $(11)$. This "double listing" is required to accommodate the waveform indexing scheme to produce the proper 32 waveforms. The representation is largely notional and is used to conceptualize the relationships between FQPSK and SOQSPK.

The asymptotic performance of maximum likelihood detection of FQPSK has been analyzed by Simon [14]. In concept, maximum likelihood detection of FQPSK organizes the outputs of 32 matched filters (one filter matched to each of the 32 possible transmitted waveforms) in a trellis and performs maximum likelihood sequence estimation. The standard union bound composed of pairwise error probabilities is used to quantify the bit error rate performance of this modulation. The minimum distance error events span three trellis states. Over this span, every trellis state is reachable by every trellis state via two paths. Thus there are $32 \times 32 \times 2=2048$ such paths over three steps. Each of these paths has one competing path associated with it that contributes a single bit error. 1024 of these path pairs are separated by a normalized Euclidean distance of 1.56 and 1024 of these path pairs are separated by a normalized Euclidean distance of 2.59. As such, the probability of bit error is well approximated by

$$
P_{b} \approx 1 \times \frac{1024}{2048} Q\left(\sqrt{1.56 \frac{E_{b}}{N_{0}}}\right)+1 \times \frac{1024}{2048} Q\left(\sqrt{2.59 \frac{E_{b}}{N_{0}}}\right)
$$

(Why the coefficients are expressed this way will become evident in Section III.) Using this expression, $P_{b}=10^{-5}$ is achieved at $E_{b} / N_{0}=10.32 \mathrm{~dB}$. 


\section{Symbol-by-Symbol Detection}

SOQPSK-TG and FQPSK-JR are considered to be interoperable because of their essentially identical bandwidth and similar bit error rate performance with symbol-by-symbol detection using an integrateand-dump detection filter. Using an unshaped offset QPSK detector with FQPSK (and its variants) is natural since FQPSK is defined as an offset modulation with data dependent pulse shapes. The use of this detection technique with SOQPSK-TG is motivated by the well established connection between CPM with modulation index $h=1 / 2$ and offset QPSK [15]-[18]. Symbol-by-symbol detection has been thoroughly investigated for SOQPSK-TG by Geoghegan [10] and for FQPSK by Simon [14]. Our own simulation results are shown in [19, Figure 2] where we see that $\Delta_{\mathrm{S}} \approx 2.0 \mathrm{~dB}$ and $\Delta_{\mathrm{F}} \approx 2.2 \mathrm{~dB}$. Symbol-by-symbol detection with better detection filters has also been investigated for SOQPSK-TG in [10] and for FQPSK in [14]. The XTCQM representations for both modulations can be used to define detection filters for use with a symbol-by-symbol detector as explained in Appendix A. The bit error rate performance of SOQPSK-TG and FQPSK-JR using the improved detection filter is also plotted in [19, Figure 2]. Observe that the improved performance reduces $\Delta_{\mathrm{S}}$ to about $1.5 \mathrm{~dB}$ and $\Delta_{\mathrm{F}}$ to about $1.6 \mathrm{~dB}$. The performance of this approach falls well short of that of maximum likelihood detection since symbol-by-symbol detection ignores the memory inherent in the waveforms. The fact that these losses are still significant motivates the search for common detectors that perform better than the symbol-by-symbol detector.

\section{COMmon XTCQM Detector}

A generic maximum-likelihood XTCQM detector is illustrated in Figure 3. The in-phase component of the noisy received waveform is filtered by a bank of filters matched to the $N_{\mathrm{X}}$ possible in-phase waveforms. Likewise, the quadrature component of the received waveform is filtered by a bank of filters matched to the possible quadrature waveforms. These matched filter outputs are sampled, once per symbol, and used by a maximum likelihood sequence estimator operating on a trellis with $2 N_{\mathrm{X}}$ states. For FQPSK and FQPSK-JR, $N_{\mathrm{X}}=16$.

In order to formulate such a detector for SOQPSK-TG, an XTCQM representation of SOQPSK-TG is needed. This representation, developed in Appendix A, is of the form

$$
s_{\mathbf{S}}(t)=\sum_{k} I_{\mathbf{S}}\left(t-k T_{s} ; a_{2 k+1}, \ldots, a_{2 k-9}\right)+j Q_{\mathbf{S}}\left(t-k T_{s} ; a_{2 k+1}, \ldots, a_{2 k-9}\right) .
$$

This representation consists of 1024 in-phase waveforms $I_{\mathrm{S}}(t)$ and 1024 quadrature waveforms $Q_{\mathrm{S}}(t)$ indexed by a sliding window that is 11 bits wide and strides through the bits 2 at a time. The XTCQM 
representation of SOQPSK-TG requires 2048 complex waveforms and is an exact representation of this modulation. The maximum-likelihood XTCQM detector is that of Figure 3 with $N_{\mathrm{X}}=1024$. Since this detector performs maximum likelihood detection, the bit error rate performance of this detector is given by (10). It will prove helpful below to re-express the probability of error for this detector in terms of error events on the waveform trellis. The minimum distance error event spans 6 trellis stages and produces a single bit error. Every state is reachable by every state over this interval via two paths. Thus, there are a total of $2048 \times 2048 \times 2=2^{23}$ possible paths to consider. Each path has a single competing path. Half of these path pairs are separated by a normalized Euclidean distance of 1.60 and the other half are separated by a normalized Euclidean distance of 2.58 . Thus the probability of error is

$$
P_{b} \approx \frac{1 \times 2^{22}}{2^{23}} Q\left(\sqrt{1.60 \frac{E_{b}}{N_{0}}}\right)+\frac{1 \times 2^{22}}{2^{23}} Q\left(\sqrt{2.58 \frac{E_{b}}{N_{0}}}\right) .
$$

Since the number of matched filters and the number of trellis states is different for FQPSK-JR and SOQPSK-TG, the forgoing formulation does not permit Figure 3 to operate as a common detector for both modulations. This issue is resolved by identifying a set of 32 waveforms suitable for use with both FQPSK-JR and SOQPSK-TG in Figure 3. The first step is to reduce the number of waveforms required to represent SOQPSK-TG to 32. This is achieved using the averaging technique described in Appendix A to produce

$$
s_{\mathrm{S}}(t) \approx \sum_{k} \tilde{I}_{\mathrm{S}}\left(t-k T_{s} ; a_{2 k-2}, \ldots, a_{2 k-6}\right)+j \tilde{Q}_{\mathrm{S}}\left(t-k T_{s} ; a_{2 k-2}, \ldots, a_{2 k-6}\right) .
$$

The in-phase waveform $\tilde{I}_{\mathrm{S}}(t ; \cdot)$ is drawn from a set of 16 waveforms while the quadrature waveform $\tilde{Q}_{\mathrm{S}}(t ; \cdot)$ is drawn from a different set of 16 waveforms. The total number of complex-valued waveforms is 32 and 5 bits are used to select the waveform. Note that with a simple redefinition of the bit indexes, the representation (16) is identical in form to the XTCQM representation of FQPSK-JR given by (12).

Since the sets of waveforms from which the waveforms $I_{\mathrm{F}}(t ; \cdot)$ and $\tilde{I}_{\mathrm{S}}(t ; \cdot)$ are drawn are different, a common set must be identified in order to produce a common detector of the form shown in Figure 3. The common waveforms define the matched filters and trellis connections. Three possibilities were explored: 1) the FQPSK-JR waveforms $I_{\mathrm{F}}(t ; \cdot)$ and $\left.Q_{\mathrm{F}}(t ; \cdot) ; 2\right)$ the SOQPSK-TG waveforms $\tilde{I}_{\mathrm{S}}(t ; \cdot)$ and $\tilde{Q}_{\mathrm{S}}(t ; \cdot)$; and 3) average waveforms

$$
\begin{aligned}
I_{\text {avg }}(t ; \cdot) & =\frac{I_{\mathrm{F}}(t ; \cdot)+\tilde{I}_{\mathrm{S}}(t ; \cdot)}{2} \\
Q_{\text {avg }}(t ; \cdot) & =\frac{Q_{\mathrm{F}}(t ; \cdot)+\tilde{Q}_{\mathrm{S}}(t ; \cdot)}{2} .
\end{aligned}
$$


A number of other possibilities could be envisioned (e.g., waveforms that minimize the average squared error). However, the performance results, summarized below, show that a detector based on the average waveforms is on the order of $1 / 10$ of a $\mathrm{dB}$ from optimum. This suggests there is very little to be gained by using waveforms based on more elaborate criteria.

Since the set of waveforms used by the detector is different from the set of waveforms used by the modulator, the mismatched receiver analysis technique, described in [13], [20], [21], can be used to evaluate the performance of each of these options. Due to space limitations, only the performance of the common detector based on the average waveforms is reported here since it was the best of the three.

When FQPSK-JR is produced by the modulator, the mismatch is a result of the fact that the detector's model for the transmitted signal is based on the 32 waveforms defined by (17) rather than on the actual 32 waveforms. Each error event included in (13) involves a pair of waveforms defined by bit sequences $\mathbf{a}_{1}$ and $\mathbf{a}_{2}$. Let $s\left(t ; \mathbf{a}_{1}\right)$ and $s\left(t ; \mathbf{a}_{2}\right)$ represent the corresponding signals produced by the transmitter and let $\tilde{s}\left(t ; \mathbf{a}_{1}\right)$ and $\tilde{s}\left(t ; \mathbf{a}_{2}\right)$ represent the corresponding signal used by the detector based on its set of waveforms. The probability of the error event is

$$
Q\left(\sqrt{\tilde{d}^{2} \frac{E_{b}}{N_{0}}}\right)
$$

where

$$
\tilde{d}=\sqrt{\frac{1}{2 E_{b}}} \frac{d_{1}-d_{2}}{\sqrt{d_{3}}}
$$

and

$$
\begin{aligned}
& d_{1}=\int_{R}\left|s\left(t ; \mathbf{a}_{1}\right)-\tilde{s}\left(t ; \mathbf{a}_{2}\right)\right|^{2} d t \\
& d_{2}=\int_{R}\left|s\left(t ; \mathbf{a}_{1}\right)-\tilde{s}\left(t ; \mathbf{a}_{1}\right)\right|^{2} d t \\
& d_{3}=\int_{R}\left|\tilde{s}\left(t ; \mathbf{a}_{1}\right)-\tilde{s}\left(t ; \mathbf{a}_{2}\right)\right|^{2} d t .
\end{aligned}
$$

The 1024 pairs of sequences associated with the minimum distance error event in (13) produce a set of 1024 modified distances denoted $\tilde{d}_{l}$ (for $l=0, \ldots$ 1023) consisting of 128 unique values that range from 1.41 to 1.70 . In the same way, the 1024 pairs of sequences associated with the error event quantified by the second term in (13) produce a set of 1024 modified distances $\tilde{d}_{m}$ (for $m=0, \ldots 1023$ ) with 128 unique values ranging from 2.42 to 2.76 . The probability of error is

$$
P_{b} \approx \frac{1}{2048} \sum_{l=0}^{1023} Q\left(\sqrt{\tilde{d}_{l}^{2} \frac{E_{b}}{N_{0}}}\right)+\frac{1}{2048} \sum_{m=0}^{1023} Q\left(\sqrt{\tilde{d}_{m}^{2} \frac{E_{b}}{N_{0}}}\right) .
$$


A plot of this expression, along with computer simulations, are shown in Figure 4. This detector achieves $P_{b}=10^{-5}$ at $E_{b} / N_{0}=10.37 \mathrm{~dB}$. Thus, $\Delta_{\mathrm{F}}=0.05 \mathrm{~dB}$.

When SOQPSK-TG is produced by the modulator, the mismatch is a consequence of the fact that the detector uses only 32 waveforms (the full representation requires 2048 waveforms). As before, all 32 trellis states can be reached by all the trellis states via two paths over three trellis stages. These $32 \times 32 \times 2=2048$ trellis paths correspond to $2^{3} \times 2048 \times 2^{3}=2^{17}$ sequences that the modulator is capable of producing. (Recall that the averaging process eliminated three bits from each end of the 11-bit sequence that defined the full SOQPSK-TG waveforms.) Pairing each of the possible transmitted paths with the corresponding trellis path using the modified distance measure (18) produces a set of $2^{16}$ distances denoted $\tilde{d}_{l}^{2}$ for $l=0,1, \ldots 2^{16}-1$ ranging from 1.40 to 1.81 that correspond to the error events quantified by the first term in (15) and a set of $2^{16}$ distances denoted $\tilde{d}_{m}^{2}$ for $m=0,1, \ldots 2^{16}-1$ ranging from 2.45 to 2.71 that correspond to the error events quantified by the first term in (15). Thus the probability of error is approximated by

$$
P_{b} \approx \frac{1}{2^{17}} \sum_{l=0}^{2^{16}-1} Q\left(\sqrt{\tilde{d}_{l}^{2} \frac{E_{b}}{N_{0}}}\right)+\frac{1}{2^{17}} \sum_{m=0}^{2^{16}-1} Q\left(\sqrt{\tilde{d}_{m}^{2} \frac{E_{b}}{N_{0}}}\right)
$$

A plot of this expression, along with computer simulations, are shown in Figure 4. This detector achieves $P_{b}=10^{-5}$ at $E_{b} / N_{0}=10.35 \mathrm{~dB}$. Thus, $\Delta_{\mathrm{S}}=0.14 \mathrm{~dB}$.

The common XTCQM detector has the stucture shown in Figure 3. As explained above, there are $N_{\mathrm{X}}=32$ complex waveforms in the common XTCQM representation of SOQPSK-TG and FQPSK-JR. However, there are only 16 unique inphase waveforms (each is repeated once). In addition, these 16 waveforms consist of 8 waveforms and their negatives. The same is true for the quadrature waveforms. Furthermore, the 8 quadrature waveforms are shifted versions of the 8 inphase waveforms. By exploiting these symmetries, one can reduce $N_{\mathrm{X}}$ to 8 in Figure 3. Consequently the common XTCQM detector has 16 real valued length $2 T_{b}$ matched filters and a 16 state trellis as described by Simon in [14], [22].

\section{Common CPM Detector}

Another candidate for the common detector is the CPM detector. SOQPSK-TG is defined as a CPM, as explained in Section II-A. In order to formulate a common CPM detector, a CPM representation of FQPSK-JR is needed. This representation is developed in Appendix B and is of the form given by (3) (4) for SOQPSK with the same constrained ternary alphabet (5) and a length-2T frequency pulse $g_{\mathrm{F}}(t)$ given by (44). 
The two most common approaches for detecting CPM signals are the detector based on the traditional complex exponential representation of CPM [13] and the detector based on the PAM decomposition of CPM [23]-[29]. In the following we explore common CPM detectors based on both of those approaches.

\section{A. Common Detector Based on Complex Exponential Representation}

With FQPSK-JR represented (approximately) as a CPM signal, we can develop a common CPM detector for SOQPSK-TG and FQPSK-JR. The structure for the maximum likelihood detector for CPM with a length- $L$ frequency pulse and ternary symbols, whose relationship to the binary symbols is given by (5), is illustrated in Figure 5. The complex-baseband signal is processed by a bank of $2^{L+1}-1$ length- $T_{b}$ matched filters [30] that correlate the received signal with the possible waveforms defined by the correlative state vector. The matched filter (or correlator) outputs are sampled every $T_{b}$ seconds in synchronism with the ternary symbol boundaries and processed by a maximum likelihood sequence estimator operating on a trellis with $4 \times 2^{L-1}$ states. (Note that with the ternary alphabet (5), there are 4 phase states.)

In order for this structure to operate as a common detector, the representation for both waveforms must be based on a common frequency pulse. Since $L=8$ for SOQPSK-TG and $L=2$ for the FQPSK-JR approximation, the first step is to truncate the SOQPSK-TG frequency pulse $g_{\mathbf{S}}(t)$ to span $2 T_{b}$. This is a common technique for reducing the complexity of partial response CPM [20]. The next step is to identify a suitable frequency pulse upon which the detector can be based. As with the case for the common XTCQM detector, several options could be envisioned. The three explored in this work are the truncated SOQPSK-TG frequency pulse

$$
g_{1}(t)=\left\{\begin{array}{ll}
g_{\mathrm{S}}(t) & 3 T_{b} \leq t \leq 5 T_{b} \\
0 & \text { otherwise }
\end{array},\right.
$$

the frequency pulse of the CPM approximation for FQPSK-JR

$$
g_{2}(t)=g_{\mathrm{F}}(t),
$$

and the average of the first two

$$
g_{3}(t)=\frac{g_{1}(t)+g_{2}(t)}{2} .
$$

All three of these pulses have length $L=2$ bit times. There are seven pairs of $\left(\alpha_{n-1}, \alpha_{n}\right)$ that the receiver can encounter ${ }^{3}$, so seven length- $T_{b}$ complex matched filters are required. Exploiting the symmetries of cosine and sine, the filterbank may be implemented using 12 real-valued, length- $T_{b}$ filters and two integrateand-dump filters.

\footnotetext{
${ }^{3}$ There are a total of $3^{2}=9$ different combinations of two ternary symbols, but the combinations $(-1,+1)$ and $(+1,-1)$ are not allowed as a result of the constraints that (5) places on the sequence of ternary symbols.
} 
The mismatched receiver analysis used in Section III can be used here to determine which of the three candidates for the common detector is the best option. In all cases, the error event given by (9) is the one of interest. The probability of bit error for each case is of the form

$$
P_{b} \approx \frac{1}{L_{1}} \sum_{l=0}^{L_{2}-1} Q\left(\sqrt{\tilde{d}_{l}^{2} \frac{E_{b}}{N_{0}}}\right)+\frac{1}{M_{1}} \sum_{m=0}^{M_{2}-1} Q\left(\sqrt{\tilde{d}_{m}^{2} \frac{E_{b}}{N_{0}}}\right) .
$$

First consider the case where the common detector based on FQPSK-JR is transmitted and the detector is based in the frequency pulse $g_{1}(t)$. The constraints on the error event are described as follows: Returning to the sequence (8), pairs of sequences for which

$$
\begin{array}{lll}
a_{k-3}=a_{k+3} & & a_{k-3}=-a_{k+3} \\
a_{k-1}=a_{k+1} & \text { or } & a_{k-1}=a_{k+1} \\
a_{k-3}=a_{k-1} & & a_{k-3}=a_{k-1}
\end{array}
$$

produce pairs of 32 waveforms separated by modified distances ranging from 1.41 to 1.70 . Pairs of sequences for which

$$
\begin{array}{lll}
a_{k-3}=a_{k+3} & & a_{k-3}=-a_{k+3} \\
a_{k-1}=-a_{k+1} & \text { or } & a_{k-1}=-a_{k+1} \\
a_{k-3}=a_{k-1} & a_{k-3}=a_{k-1}
\end{array}
$$

produce pairs of 32 waveforms separated by modified distances ranging from 2.45 to 2.72 . The probability of bit error is given by (27) with $L_{1}=M_{1}=64$ and $L_{2}=M_{2}=32$. The results for the detectors based on the other two frequency pulses are summarized as follows:

- When the detector is based on the frequency pulse $g_{2}(t)$, the forgoing analysis applies except that the first set of sequence pairs produces 32 pairs of waveforms separated by modified distances ranging from 1.54 to 1.58 and the second set of sequence pairs produces 32 pairs of waveforms separated by modified distances ranging from 2.57 to 2.61 .

- When the detector is based on the frequency pulse $g_{3}(t)$, the first set of sequence pairs produces 32 pairs of waveforms separated by modified distances ranging from 1.49 to 1.62 and the second set of sequence pairs produces 32 pairs of waveforms separated by modified distances ranging from 2.52 to 2.65 .

When SOQPSK-TG is transmitted, the sequence (8) needs to be extended to account for the fact that the transmitter is based on an $L=8$ frequency pulse and the detector is based on an $L=2$ frequency 
pulse. The binary symbol sequence pairs producing the dominant error events are too tedious to describe here. The end result is that the probability of error is well approximated by (27) with $L_{1}=M_{1}=256$ and $L_{2}=M_{2}=128$.

- When the detector is based on the frequency pulse $g_{1}(t)$, the modified distances in the first sum of (27) range from 1.12 to 2.11 and the modified distances in the second sum range from 2.32 to 2.81 .

- When the detector is based on the frequency pulse $g_{2}(t)$, the modified distances in the first sum of (27) range from 1.26 to 1.93 and the modified distances in the second sum range from 2.29 to 2.86 .

- When the detector is based on the frequency pulse $g_{3}(t)$, the modified distances in the first sum of (27) range from 1.19 to 2.02 and the modified distances in the second sum range from 2.31 to 2.84 .

Plots of (27) for these cases are illustrated in Figure 6. Simulation results are also included to demonstrate how accurate the approximations are. The detector based on frequency pulse $g_{2}(t)$ provides the best performance for both FQPSK-JR and SOQPSK-TG. Thus the common CPM detector is one based on $g_{2}(t)$ and achieves $\Delta_{\mathrm{S}}=0.21 \mathrm{~dB}$ and $\Delta_{\mathrm{F}}=0.01 \mathrm{~dB}$.

\section{B. Common Detector Based on PAM Representation}

It is well known that CPM signals can be represented as sums of PAM signals [23], [25], [27], [29]. Since SOQPSK-TG is defined as a CPM, and with the CPM approximation of FQPSK-JR, the PAM representations of these modulations provide another option for a common detector. The PAM decomposition of CPM signals is well known to provide an alternate and often greatly simplified structure for detecting CPM signals. The PAM representation of binary CPM was first introduced by Laurent [23] and was used as the basis for simplified CPM detectors by Kaleh [24]. The representation was later extended to M-ary CPM (with M even) by Mengali and Morelli [25] and applied to the detection of CPM by Colavolpe and Raheli [26]. The non-binary multi-index case was explored by Perrins and Rice [27]. Recently the PAM decomposition for ternary CPM has been developed [29]. This PAM decomposition was applied to SOQPSK-TG in [31].

A generic block diagram of the maximum likelihood detector for CPM based on the PAM representation is illustrated in Figure 7. A bank of filters, each matched to one of the $N_{P}$ constituent PAM pulses processes the received signal at complex baseband. The filter outputs are sampled once per bit and used by a maximum likelihood sequence estimator operating on a trellis with $S_{P}$ states. In order to formulate a common PAM detector, it is necessary to approximate both SOQPSK-TG and FQPSK-JR by the same umber of PAM pulses. Suitable pulses for this approximation must also be identified. 
The PAM decomposition of SOQPSK-TG consists of representing the continuous phase signal as the sum of pulse amplitude modulated signals. Thus the nonlinear modulation is transformed into a linear modulation where the modulating symbols (the so-called pseudo-symbols) are related to the original data symbols in a nonlinear way. The PAM decomposition of SOQPSK-TG is given by

$$
s_{\mathrm{S}}(t)=\sum_{k=0}^{4373} \sum_{i} b_{k, i} c_{k, \mathrm{~S}}\left(t-i T_{b}\right)
$$

where $b_{k, i}$ are the pseudo-symbols which are a function of the data bits as explained in [29] and $c_{k, \mathrm{~S}}(t)$ are the PAM pulses for the decomposition of SOQPSK-TG. The PAM pulses have varying lengths ranging from $(L+1) T_{b}$ (which is $9 T_{b}$ in this case) to $T_{b}$.

The large number of terms in the sum in (28) is due to the length of the SOQPSK-TG frequency pulse $g_{\mathrm{S}}(t)$ and would appear to render this representation prohibatively complex. However, as is often the case with the PAM decomposition of partial response CPM, the vast majority of the PAM pulses $c_{k, \mathrm{~S}}(t)$ contain essentially no energy and hence can be neglected. In fact, $s_{\mathrm{S}}(t)$ is well approximated as the sum of only two pulses which are known as the principal pulses [31]. As a result, (28) can be rewritten as

$$
s_{\mathrm{S}}(t) \approx \sum_{i} b_{0, i} c_{0, \mathrm{~S}}\left(t-i T_{b}\right)+b_{1, i} c_{1, \mathrm{~S}}\left(t-i T_{b}\right)
$$

The principal pulses for SOQPSK-TG are plotted in Figure 8. Strictly speaking, the pulses $c_{0, \mathrm{~S}}(t)$ and $c_{1, \mathrm{~S}}(t)$ have lengths $9 T_{b}$ and $8 T_{b}$, respectively. However, as can be seen in Figure 8, these pulses are approximately zero over most of their durations. We will exploit this observation when forming the common PAM detector below.

The PAM decomposition for FQPSK-JR is

$$
s_{\mathrm{F}}(t)=\sum_{k=0}^{5} \sum_{i} b_{k, i} c_{k, \mathrm{~F}}\left(t-i T_{b}\right) .
$$

There are six pulses in the PAM representation of FQPSK-JR, but four of them contain negligible energy, so the PAM decomposition of FQPSK-JR is well approximated as

$$
s_{\mathrm{F}}(t) \approx \sum_{i} b_{0, i} c_{0, \mathrm{~F}}\left(t-i T_{b}\right)+b_{1, i} c_{1, \mathrm{~F}}\left(t-i T_{b}\right) .
$$

The principal pulses $c_{0, \mathrm{~F}}(t)$ and $c_{1, \mathrm{~F}}(t)$ for FQPSK-JR have lengths $3 T_{b}$ and $2 T_{b}$, respectively and are shown in Figure 8.

The trellis for the SOQPSK-TG based PAM detector is identical to the trellis for the FQPSK-JR-based PAM detector. This is because the relationship between the pseudo-symbols and the data bits is the same 
for both modulations. The trellis has $S_{P}=4$ states and is decribed in [31]. This trellis is the obvious choice for the common detector trellis.

The remaining task in formulating the common PAM detector is the determination of the common matched filters. As explained above, the PAM pulses for SOQPSK-TG are much longer than those for FQPSK-JR. However, as also explained above, the SOQPSK-TG PAM pulses are essentially equal to zero over the majority of their durations. In fact, the first and second principal SOQPSK-TG pulses are very well approximated as being nonzero over lengths of $3 T_{b}$ and $2 T_{b}$, respectively, which are the lengths of the principal FQPSK-JR PAM pulses. Consequently, we consider the following options for the PAM pulses for the common PAM detector: Option 1, the truncated SOQPSK-TG based PAM pulses

$$
c_{0,1}(t)=\left\{\begin{array}{ll}
c_{0, \mathrm{~S}}(t) & 3 T_{b} \leq t \leq 6 T_{b} \\
0 & \text { otherwise }
\end{array} \quad c_{1,1}(t)=\left\{\begin{array}{ll}
c_{1, \mathrm{~S}}(t) & 4 T_{b} \leq t \leq 6 T_{b} \\
0 & \text { otherwise }
\end{array},\right.\right.
$$

Option 2, the FQPSK-JR based PAM pulses

$$
c_{0,2}(t)=c_{0, \mathrm{~F}}(t) \quad c_{1,2}(t)=c_{1, \mathrm{~F}}(t),
$$

and Option 3, pulses which are the average of those two

$$
c_{0,3}(t)=\frac{c_{0,1}(t)+c_{0,2}(t)}{2} \quad c_{1,3}(t)=\frac{c_{1,1}(t)+c_{1,2}(t)}{2}
$$

All of the options listed above have length $3 T_{b}$ and $2 T_{b}$ for the two PAM pulses. As a result, the common PAM detector has 2 real-valued matched filters which are the equivalent of 5 real-valued length $T_{b}$ matched filters. The common PAM detector is a special case of the detector of Figure 7 with $N_{P}=2$ and $S_{P}=4$.

A mismatched receiver analysis can be used to determine which set of the candidate PAM pulses listed above is the best option for the common PAM detector. The analysis for the PAM detector differs from the analysis for the XTCQM or CPM detector because the distance measure given by (18) does not apply as explained in [28]. The modified distance measure $d$ that is appropriate for PAM detectors along with the resulting expression for the probability of bit error is given in [28]. We apply this analysis to the candidates for the common PAM detector here.

First consider the case when the transmitted signal is SOQPSK-TG. With the PAM detector based on option 1, there are 32 sequence pairs with normalized squared distances ranging from 1.43 to 1.74 and 32 ranging from 2.21 to 2.94 . With option 2 there are 32 sequence pairs with normalized squared distances ranging from 1.42 to 1.75 and 32 ranging from 2.23 to 2.91 . With option 3 there are 32 sequence pairs with normalized squared distances ranging from 1.42 to 1.75 and 32 ranging from 2.22 to 2.93 . 
Next consider the case when FQPSK-JR is transmitted. With option 1 there are 32 pairs of sequences with normalized squared distances ranging from 1.37 to 1.75 and 32 ranging from 2.36 to 2.81. With option 2 there are 32 ranging from 1.38 to 1.73 and 32 ranging from 2.38 to 2.77, and with option 3 there are 32 ranging from 1.38 to 1.74 along with 32 ranging from 2.37 to 2.79 . Because the normalized squared distances are so similar, there is very little difference in the probability of bit error among the three options for the common PAM detector. In fact, when SOQPSK-TG it the transmitted modulation the three options vary by less than $0.02 \mathrm{~dB}$ at $P_{b}=10^{-5}$ and when FQPSK-JR is the transmitted modulation they vary by less than $0.01 \mathrm{~dB}$ at that same $P_{b}$. The PAM detector based on Option 1 , the truncated SOQPSK-TG PAM pulses, is very slightly better than the other options, so that detector is chosen as the common PAM detector. The probability of bit error for this detector is plotted in Figure 9. With this common PAM detector $\Delta_{\mathrm{S}}=0.11 \mathrm{~dB}$ and $\Delta_{\mathrm{F}}=0.09 \mathrm{~dB}$. Computer simulations confirm the mismatched analysis and are also plotted in Figure 9.

\section{Conclusions}

SOQPSK-TG and FQPSK-JR share many similarities. We have shown that both may be represented as cross-correlated trellis-coded quadrature modulations and that both may be represented as continuous phase modulations (although the CPM interpretation for FQPSK-JR is only an approximation). These common views confirm their interoperability and suggest architectures for common detectors: XTCQM, CPM, and PAM based detectors. We have shown that common detectors based on these representations produce good performance for both modulations and that the performance is a great improvement over the existing common detector based on symbol-by-symbol detection. This improvement comes at the cost of increased complexity in the detector. The attractive feature of these common detectors is that they do not require knowledge of which modulation is employed by the transmitter.

The bit error rate performance and complexity of these detectors are summarized in Table I. All three of the proposed common detectors have bit error rate performances that are fairly close to optimum. The common CPM detector has the best performance for FQPSK-JR $\left(\Delta_{\mathrm{F}}=0.01 \mathrm{~dB}\right)$ and the common PAM detector has the best performance for SOQPSK-TG $\left(\Delta_{\mathrm{S}}=0.11 \mathrm{~dB}\right)$. The overall detection efficiency loss given by the sum of $\Delta_{S}$ and $\Delta_{F}$ for the three common detectors is almost identical: for the common XTCQM detector $\Delta_{\mathrm{S}}+\Delta_{\mathrm{F}}=0.19 \mathrm{~dB}$, for the common CPM detector $\Delta_{\mathrm{S}}+\Delta_{\mathrm{F}}=0.22 \mathrm{~dB}$, and for the common PAM detector $\Delta_{\mathrm{S}}+\Delta_{\mathrm{F}}=0.20$. However, since the PAM detector has lower complexity than the other two detectors, it appears to be the best option for the common detection of SOQPSK-TG and 


\section{FQPSK-JR.}

\section{APPENDIX A \\ XTCQM REPRESENTATION OF SOQPSK-TG}

In order to obtain the XTCQM representation of SOQPSK-TG we need to determine the data dependent pulses $I_{\mathrm{S}}\left(t ; a_{n}, \ldots\right)$ and $Q_{\mathrm{S}}\left(t ; a_{n}, \ldots\right)$ for this modulation which are analogous to those for FQPSK-JR in (12). These waveforms have a duration of $2 T_{b}$, which causes the resulting XTCQM signal trellis to be time invariant ${ }^{4}$. In order to obtain length $2 T_{b}$ quadrature waveforms for SOQPSK-TG, we begin by examining the phase (4) during the interval $n T_{b} \leq t \leq(n+1) T_{b} . \phi(t, \boldsymbol{\alpha})$ during this interval can be written as

$$
\begin{aligned}
\phi(t, \boldsymbol{\alpha}) & =2 \pi h \sum_{k=-\infty}^{n-L} \alpha_{k} q_{\mathrm{s}}\left(t-k T_{b}\right)+2 \pi h \sum_{k=n-L+1}^{n} \alpha_{k} q_{\mathrm{S}}\left(t-k T_{b}\right) \\
& =\frac{\pi}{2} \sum_{k=-\infty}^{n-L} \alpha_{k}+\pi \sum_{k=n-L+1}^{n} \alpha_{k} q_{\mathrm{s}}\left(t-k T_{b}\right) \quad n T_{b} \leq t \leq(n+1) T_{b} .
\end{aligned}
$$

The duration of interest can be extended from $(n+1) T_{b}$ to $(n+2) T_{b}$ by extending the sum for the correlative state vector to produce

$$
\phi(t ; \boldsymbol{\alpha})=\frac{\pi}{2} \sum_{l=-\infty}^{n-L} \alpha_{l}+\pi \sum_{l=n-L+1}^{n+1} \alpha_{l} q_{\mathrm{S}}\left(t-l T_{b}\right) \quad n T_{b} \leq t \leq(n+2) T_{b}
$$

where $n$ is now constrained to be even. We make this constraint explicit by setting $n=2 k$. Inserting (5) into (36), results in

$$
\phi\left(t ; \mathbf{a}_{2 k}\right)=\theta_{2 k}+\pi \sum_{i=2 k-L+1}^{2 k+1} \frac{(-1)^{i+1} a_{i-1}\left(a_{i}-a_{i-2}\right)}{2} q_{\mathrm{S}}\left(t-i T_{b}\right)
$$

where $\mathbf{a}_{2 k}=\left[\begin{array}{llll}a_{2 k-L-1} & a_{2 k-L} & \cdots & a_{2 k+1}\end{array}\right]$ and $\theta_{2 k}=\frac{\pi}{2} \sum_{i=-\infty}^{2 k-L} \alpha_{i}$ is the phase state. With $L=8 T_{b}$ there are 9 terms in the sum in (37) and 11 bits that contribute to $\phi\left(t, \mathbf{a}_{2 k}\right)$ during the interval $2 k T_{b} \leq$ $t \leq(2 k+2) T_{b}$. The phase state $\theta_{2 k}$ does not introduce a dependency on any additional bits. Therefor the number of waveforms is determined soley by the number of bits that contribute to the sum in (37). As a result, 2048 complex waveforms are needed to exactly represent SOQPSK-TG as an XTCQM. The I and Q waveforms are given by

$$
I_{\mathbf{S}}\left(t ; \mathbf{a}_{2 k}\right)=\cos \left(\phi\left(t ; \mathbf{a}_{2 k}\right)\right), \quad Q_{\mathrm{S}}\left(t ; \mathbf{a}_{2 k}\right)=\sin \left(\phi\left(t ; \mathbf{a}_{2 k}\right)\right)
$$

\footnotetext{
${ }^{4}$ Aulin's quadrature representation of CPM has waveforms with a duration of $T_{b}$ and the resulting signal trellis is time varying [32]. Rimoldi incorporated a tilted phase into Aulin's length $T_{b}$ representation [33] to obtain a time invariant trellis. Simon took a different approach to obtain a quadrature representation with a time invariant trellis: he extended the duration of the waveforms to $2 T_{b}$ [9]. Our quadrature representation of SOQPSK-TG is a combination of Aulin's approach and Simon's approach. It has a time invariant trellis due to the fact that the waveforms have a duration of $2 T_{b}$ similar to Simon but it does not have an encoder separate from a waveform mapper as Simon's representation does.
} 
Then the XTCQM representation of SOQPSK-TG can be expressed as

$$
s_{\mathrm{S}}(t)=\sum_{k} I_{\mathrm{S}}\left(t-k T_{s} ; \mathbf{a}_{2 k}\right)+j Q_{\mathrm{S}}\left(t-k T_{s} ; \mathbf{a}_{2 k}\right)
$$

This shows that even though SOQPSK-TG is defined as a constrained ternary CPM, it can also be viewed as a XTCQM consisting of 2048 waveforms. This view suggests an alternate form for the optimal detector: an XTCQM detector.

As explained in Section III, the common detector requires a representation for SOQPSK that uses 32 waveforms instead of the 2048 required by (39). The number of waveforms required by the XTCQM representation of SOQPSK-TG can be reduced by averaging the waveforms that differ in the first and last bits. These are the waveforms that are most similar. (This technique was used by Simon [22] to reduce the number of waveforms required to represent FQPSK.) This averaging technique is illustrated for the inphase waveforms $I_{\mathrm{S}}(t ; \cdot)$ below. Application to the quadrature waveforms $Q_{\mathrm{S}}(t ; \cdot)$ is straight forward.

The number of inphase waveforms is reduced from 2048 to 512 using

$$
\begin{aligned}
I_{\mathrm{S}, 512}\left(t ; a_{2 k-8}, \ldots, a_{2 k}\right)=\frac{1}{4} I_{\mathrm{S}}( & \left.;-1, a_{2 k-8}, \ldots, a_{2 k},-1\right)+\frac{1}{4} I_{\mathrm{S}}\left(t ;-1, a_{2 k-8}, \ldots, a_{2 k},+1\right) \\
& +\frac{1}{4} I_{\mathrm{S}}\left(t ;+1, a_{2 k-8}, \ldots, a_{2 k},-1\right)+\frac{1}{4} s_{\mathrm{S}}\left(t ;+1, a_{2 k-8}, \ldots, a_{2 k},+1\right) .
\end{aligned}
$$

Performing the same averaging process on the 512 waveforms results in the 128 waveforms given by

$$
\begin{array}{r}
I_{\mathrm{S}, 128}\left(t ; a_{2 k-7}, \ldots, a_{2 k-1}\right)=\frac{1}{4} I_{\mathrm{S}, 512}\left(t ;-1, a_{2 k-7}, \ldots, a_{2 k-1},-1\right)+\frac{1}{4} I_{\mathrm{S}, 512}\left(t ;-1, a_{2 k-7}, \ldots, a_{2 k-1},+1\right) \\
+\frac{1}{4} I_{\mathrm{S}, 512}\left(t ;+1, a_{2 k-7}, \ldots, a_{2 k-1},-1\right)+\frac{1}{4} I_{\mathrm{S}, 512}\left(t ;+1, a_{2 k-7}, \ldots, a_{2 k-1},+1\right)
\end{array}
$$

and averaging those waveforms results in the 32 waveforms given by

$$
\begin{array}{r}
I_{\mathrm{S}, 32}\left(t ; a_{2 k-6}, \ldots, a_{2 k-2}\right)= \\
+\frac{1}{4} I_{\mathrm{S}, 128}\left(t ;-1, a_{2 k-6}, \ldots, a_{2 k-2},-1\right)+\frac{1}{4} I_{\mathrm{S}, 128}\left(t ;-1, a_{2 k-6}, \ldots, a_{2 k-2},+1\right) \\
+\frac{1}{4} I_{\mathrm{S}, 128}\left(t ;+1, a_{2 k-6}, \ldots, a_{2 k-2},-1\right)+\frac{1}{4} I_{\mathrm{S}, 128}\left(t ;+1, a_{2 k-6}, \ldots, a_{2 k-2},+1\right) .
\end{array}
$$

The waveforms $I_{\mathrm{S}, 32}(t ; \cdot)$ and $Q_{\mathrm{S}, 32}(t ; \cdot)$ are the waveforms $\tilde{I}_{\mathrm{S}}(t ; \cdot)$ and $\tilde{Q}_{\mathrm{S}}(t ; \cdot)$ in $(16)$.

Note that the averaging process can be continued until only two waveforms remain. It turns out to be a single length- $2 T_{b}$ waveform and its negative. This waveform can be averaged with the corresponding average of the FQPSK-JR waveforms to produce a common waveform for use by a common symbol-bysymbol detector. The bit error rate of such a detector was discussed in Section II-C. 


\section{APPENDIX B \\ CPM APPROXIMATION OF FQPSK-JR}

The CPM approximation of FQPSK-JR is obtained by determining the phase pulse $q_{\mathrm{F}}(t)$ as a function of the XTCQM waveforms for FQPSK-JR. The length of $q_{\mathrm{F}}(t)$ is $L=2$ because the waveforms are defined over a two bit interval. The phase of the signal as it transitions from one constellation point to an adjacent point determines $q_{\mathrm{F}}(t)$.

For example, consider the case where the initial phase state is $\pi / 4$ and $\alpha_{n}=-1$. In that case the I and Q waveforms $\left(I_{\mathrm{F}}(t ; \cdot)\right.$ and $Q_{\mathrm{F}}(t ; \cdot)$, respectively) are given by ${ }^{5}$

$$
I_{\mathrm{F}}(t ; \cdot)=\sqrt{1-A^{2} \cos ^{2}\left(\frac{\pi t}{2 T_{b}}\right)} \quad Q_{\mathrm{F}}(t ; \cdot)=-A \sin \left(\frac{\pi\left(t-T_{b}\right)}{2 T_{b}}\right)
$$

for $0 \leq t \leq 2 T_{b}$. The phase pulse in this case is given by

$$
q_{\mathrm{F}}(t)=\tan ^{-1}\left[\frac{-A \sin \left(\frac{\pi\left(t-T_{b}\right)}{2 T_{b}}\right)}{\sqrt{1-A^{2} \cos ^{2}\left(\frac{\pi t}{2 T_{b}}\right)}}\right]=\tan ^{-1}\left[\frac{-A \cos \left(\frac{\pi t}{2 T_{b}}\right)}{\sqrt{1-A^{2} \cos ^{2}\left(\frac{\pi t}{2 T_{b}}\right)}}\right] .
$$

The frequency pulse $g_{\mathrm{F}}(t)$ is then the derivative of $q_{\mathrm{F}}(t)$ and is given by

$$
g_{\mathrm{F}}(t)=\frac{\frac{A \pi}{2 T_{b}} \sin \left(\frac{\pi t}{2 T_{b}}\right)}{\sqrt{1-A^{2} \cos ^{2}\left(\frac{\pi t}{2 T_{b}}\right)}}
$$

and is plotted in Figure 1 along with the phase pulse $q_{\mathrm{F}}(t)$. It is easy to show that starting with the three other phase states this approach produces the same $g_{\mathrm{F}}(t)$. The same is true for all four phase states when $\alpha_{n}=1$. When $\alpha_{n}=0$ no phase transition occurs and the $g_{\mathrm{F}}(t)$ in (44) can be assumed. Thus $g_{\mathrm{F}}(t)$ in (44) is the frequency pulse for the CPM approximation of FQPSK-JR. As explained in [19], the CPM representation of FQPSK-JR is exact when the sequence of ternary data symbols $\boldsymbol{\alpha}$ is such that a single nonzero $\alpha_{n}$ surrounded by zeros occurs but it is only approximate when two or more consecutive nonzero symbols occur, although the approximation is quite good. Since the phase shift due to non-zero values of $\alpha_{n}$ is $\pm \pi / 2$, the modulation index of the CPM approximation is $h=1 / 2$.

\footnotetext{
${ }^{5} A$ is an adjustable parameter that appears in the definitions of the 16 waveforms for FQPSK. This parameter is set to $1 / \sqrt{2}$ to produce the constant envelope FQPSK-JR.
} 


\section{REFERENCES}

[1] T. Rappaport, Wireless Communications: Principles and Practice. New York: Prentice Hall PTR, 2 nd ed., 2001.

[2] S. Horan, Introduction to PCM Telemetering Systems. CRC Press, second ed., 2002.

[3] Range Commanders Council Telemetry Group, Range Commanders Council, White Sands Missile Range, New Mexico, IRIG Standard 106-04: Telemetry Standards, 2004. [Online]. Available: http://www.jcte.jcs.mil/rcc/manuals/106-04/.

[4] P. S. Leung and K. Feher, "F-QPSK-a superior modulation technique for mobile and personal communications," IEEE Transactions on Broadcasting, vol. 39, pp. 288-294, June 1993.

[5] R. P. Jefferis, "Evaluation of constant envelope offset quadrature phase shift keying transmitters with a software based signal analyzer," in Proceedings of the International Telemetering Conference, (San Diego, CA), October 2004.

[6] T. J. Hill, "An enhanced, constant envelope, interoperable shaped offset QPSK (SOQPSK) waveform for improved spectral efficiency," in Proceedings of the International Telemetering Conference, (San Diego, CA), October 2000.

[7] K. Feher and et. al. U.S. Patents: 4,567,602; 4,339,724; 4,644,565; 5,784,402; 5,491,457. Canadian patents: 1,211,517; 1,130,871; $1,265,851$.

[8] D. I. S. Agency, "Department of defense interface standard, interoperability standard for single-access 5-kHz and 25-kHz UHF satellite communications channels," Tech. Rep. MIL-STD-188-181B, Department of Defense, March 1999.

[9] L. Li and M. K. Simon, "Performance of coded OQPSK and MIL-STD SOQPSK with iterative decoding," IEEE Transactions on Communications, vol. 52, pp. 1890-1900, November 2004.

[10] M. Geoghegan, "Optimal linear detection of SOQPSK," in Proceedings of the International Telemetering Conference, (San Diego, CA), October 2002.

[11] M. Geoghegan, "Implementation and performance results for trellis detection of SOQPSK," in Proceedings of the International Telemetering Conference, (Las Vegas, NV), October 2001.

[12] M. Geoghegan, "Bandwidth and power efficiency trade-offs of SOQPSK," in Proceedings of the International Telemetering Conference, (San Diego, CA), October 2002.

[13] J. B. Anderson, T. Aulin, and C.-E. Sundberg, Digital Phase Modulation. New York: Plenum Press, 1986.

[14] M. K. Simon and T.-Y. Yan, "Performance evaluation and interpretation of unfiltered Feher-patented quadrature phase-shift keying (FQPSK)," Telecommunications and Mission Operations Progress Report, Jet Propulsion Laboratory, May 1999. [Online]. Available: http://tmo.jpl.nasa.gov/progress_report/42-137/137C.pdf.

[15] A. Svensson and C.-E. Sundberg, "Optimum MSK-type receivers for CPM on Gaussian and Rayleigh fading channels," IEE Proceedings, pp. 480-490, August 1984.

[16] A. Svensson and C.-E. Sundberg, "Serial MSK-type detection of partial response continuous phase modulation," IEEE Transactions on Communications, vol. 33, pp. 44-52, January 1985.

[17] P. Galko and S. Pasupathy, "Linear receivers for correlatively coded MSK," IEEE Transactions on Communications, vol. 33, pp. 338347, April 1985.

[18] R. Rhodes, S. Wilson, and A. Svensson, "MSK-type reception of continuous phase modulation: Cochannel and adjacent channel interference," IEEE Transactions on Communications, vol. 35, pp. 185-193, February 1987.

[19] T. Nelson and M. Rice, "Common detectors for shaped offset QPSK (SOQPSK) and feher-patented QPSK (FQPSK)," in Proceedings of the IEEE Global Telecommunications Conference, (St. Louis, MO), November 2005.

[20] A. Svensson, C.-E. Sundberg, and T. Aulin, "A class of reduced-complexity Viterbi detectors for partial response continuous phase modulation,” IEEE Trans. Commun., vol. 32, pp. 1079-1087, Oct. 1984.

[21] E. Perrins and M. Rice, "The detection efficiency of ARTM CPM in aeronautical telemetry." submitted to IEEE Transactions on Aerospace \& Electronic Systems, 2004. [Online]. Available: https://dspace.byu.edu/handle/1877/59).

[22] M. K. Simon, Bandwidth-Efficient Digital Modulation With Application to Deep-Space Communications. New York: Wiley, 2003.

[23] P. A. Laurent, "Exact and approximate construction of digital phase modulations by superposition of amplitude modulated pulses (AMP)," IEEE Transactions on Communications, vol. 34, pp. 150-160, February 1986.

[24] G. K. Kaleh, "Simple coherent receivers for partial response continuous phase modulation," IEEE Journal on Selected Areas in Communications, vol. 7, pp. 1427-1436, December 1989.

[25] U. Mengali and M. Morelli, "Decomposition of M-ary CPM signals into PAM waveforms," IEEE Transactions on Information Theory, vol. 41, pp. 1265-1275, September 1995.

[26] G. Colavolpe and R. Raheli, "Reduced-complexity detection and phase synchronization of CPM signals," IEEE Transactions on Communications, vol. 45, pp. 1070-1079, September 1997.

[27] E. Perrins and M. Rice, "PAM decomposition of M-ary multi-h CPM," IEEE Transactions on Communications, vol. 53, pp. 2065-2075, December 2005.

[28] E. Perrins and M. Rice, "A new performance bound for PAM-based CPM detectors," IEEE Transactions on Communications, vol. 53, pp. 1688-1696, October 2005.

[29] E. Perrins and M. Rice, "PAM representation of ternary CPM." Submitted to IEEE Transactions on Communications, March 2005. See also: E. Perrins and M. Rice, "PAM Representation of Ternary Continuous Phase Modulation', Department of Electrical and Computer Engineering, Brigham Young University, February 2005. [Online]. Available: https://dspace.byu.edu/handle/1877/58.

[30] E. Perrins, R. Schober, M. Rice, and M. Simon, "Shaped-offset QPSK with multiple-bit differential detection." to appear in Proceedings of the IEEE International Communications Conference, 11-15 June 2006, Istabul, Turkey.

[31] E. Perrins and M. Rice, "Simple detectors for shaped-offset QPSK using the PAM decomposition," in Proceedings of the IEEE Global Telecommunications Conference, November 2005.

[32] T. Aulin, N. Rydbeck, and C.-E. Sundberg, "Continuous phase modulation-part II: Partial response signaling," IEEE Transactions on Communications, vol. 29, pp. 210-225, March 1981.

[33] B. E. Rimoldi, “A decomposition approach to CPM,” IEEE Transactions on Information Theory, vol. 34, pp. 260-270, March 1988. 
TABLE I

COMPARISON OF COMMON DETECTORS. NOTE THAT THE DETECTION FILTER COUNTS ARE GIVEN IN TERMS OF EQUIVALENT REAL-VALUED, LENGTH- $T_{b}$ FILTERS IN ORDER TO ALLOW A CONSISTENT COMPARISON BETWEEN THE DETECTORS.

\begin{tabular}{|l||c|c|c|c|c|}
\hline \multicolumn{7}{|c|}{$\mathbf{E}_{\mathbf{b}} / \mathbf{N}_{\mathbf{0}}(\mathbf{d B})$ for $\mathbf{P}_{\mathbf{b}}=\mathbf{1 0}^{-\mathbf{5}}$} \\
\hline & Optimal & Sym. & XTCQM & CPM & PAM \\
\hline FQPSK-JR & 10.32 & 12.0 & 10.37 & 10.33 & 10.41 \\
\hline SOQPSK-TG & 10.21 & 11.7 & 10.35 & 10.42 & 10.32 \\
\hline \hline \multicolumn{6}{|c|}{ Detection Efficiency Loss (dB) } \\
\hline$\Delta_{\mathrm{F}}$ & - & 1.6 & 0.05 & 0.01 & 0.09 \\
\hline$\Delta_{\mathrm{S}}$ & - & 1.5 & 0.14 & 0.21 & 0.11 \\
\hline \multicolumn{7}{|c|}{ Detector Complexity } \\
\hline Detection filters & - & 1 & 32 & 12 & 5 \\
\hline Trellis states & - & 1 & 16 & 8 & 4 \\
\hline
\end{tabular}

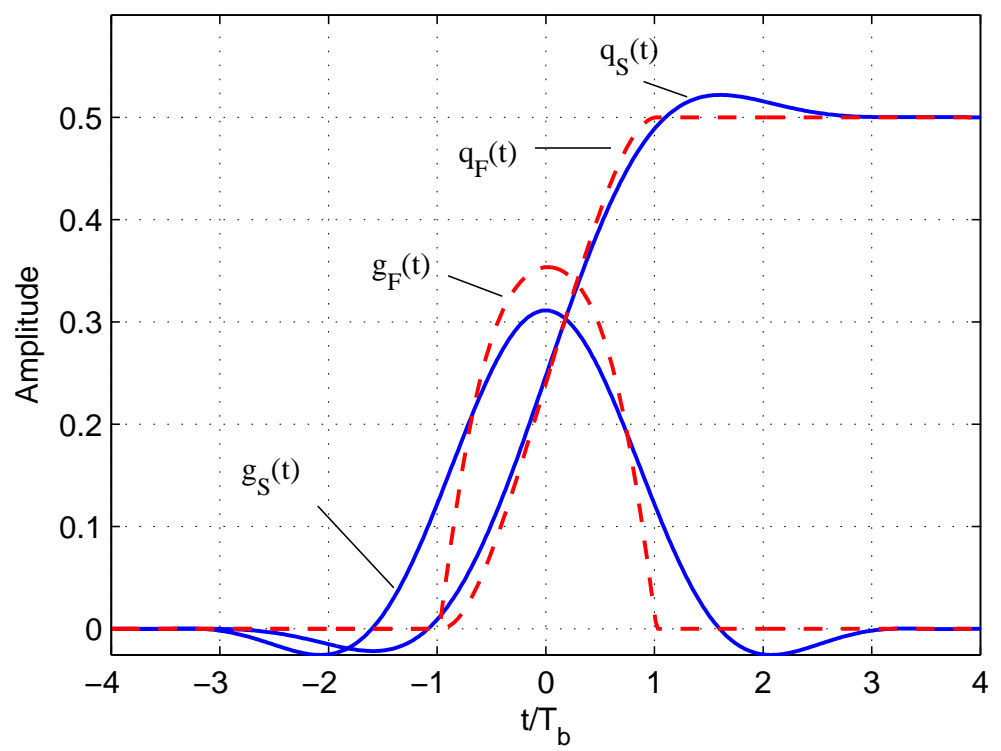

Fig. 1. The frequency pulse and phase pulse for both SOQPSK-TG $\left(g_{\mathrm{S}}(t)\right.$ and $\left.q_{\mathrm{S}}(t)\right)$ and the CPM approximation of FQPSK-JR $\left(g_{\mathrm{F}}(t)\right.$ and $q_{\mathrm{F}}(t)$ ). 


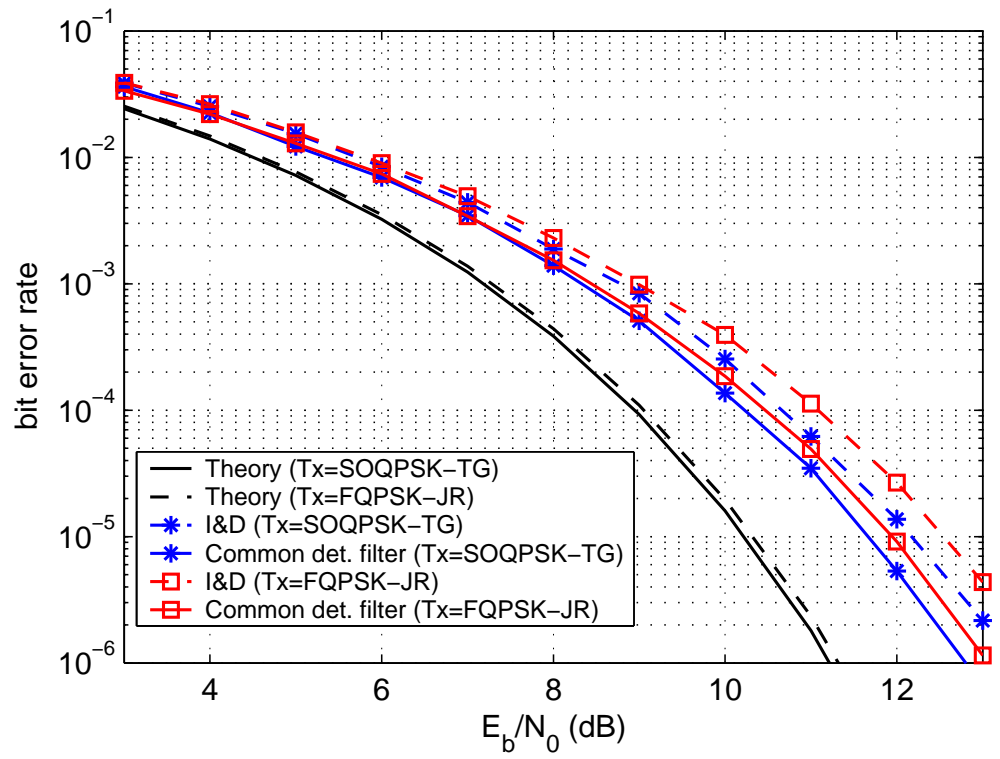

Fig. 2. Bit error rates for SOQPSK-TG and FQPSK-JR for the integrate-and-dump (I\&D) detector and the common symbol-by-symbol detector along with the theoretical curves for each modulation. For the I\&D detector $\Delta_{\mathrm{S}}=2.0 \mathrm{~dB}$ and $\Delta_{\mathrm{F}}=2.2 \mathrm{~dB}$ while the average matched filter detector has $\Delta_{\mathrm{S}}=1.5 \mathrm{~dB}$ and $\Delta_{\mathrm{F}}=1.6 \mathrm{~dB}$.

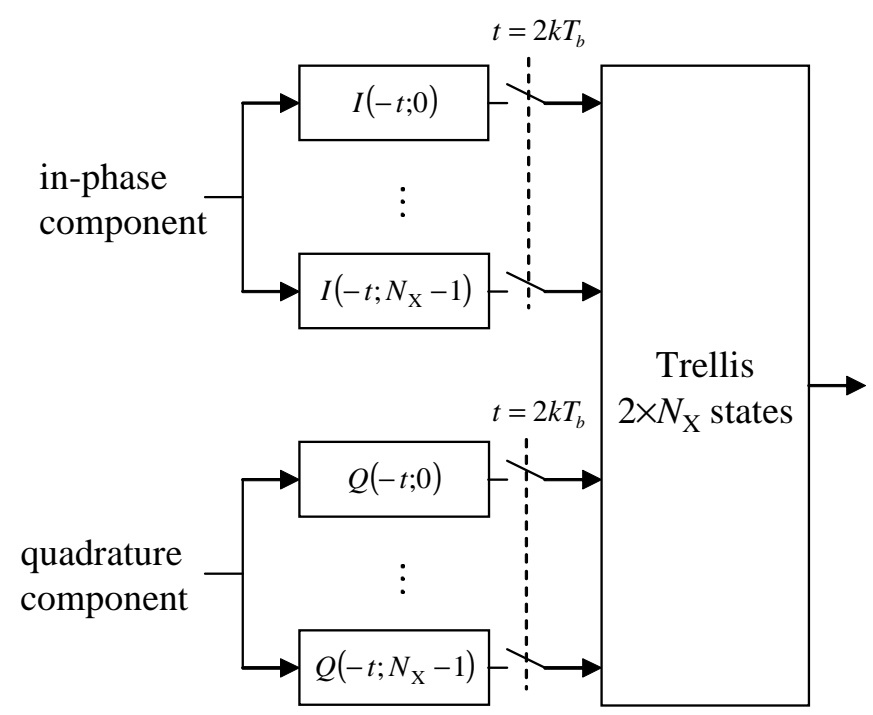

Fig. 3. Block diagram of the maximum likelihood XTCQM detector. Each filter is a real-valued filter of length $2 T_{b}$. The indexes in the filter impulse responses are the decimal equivalents of the binary symbol patterns that define the waveforms. 


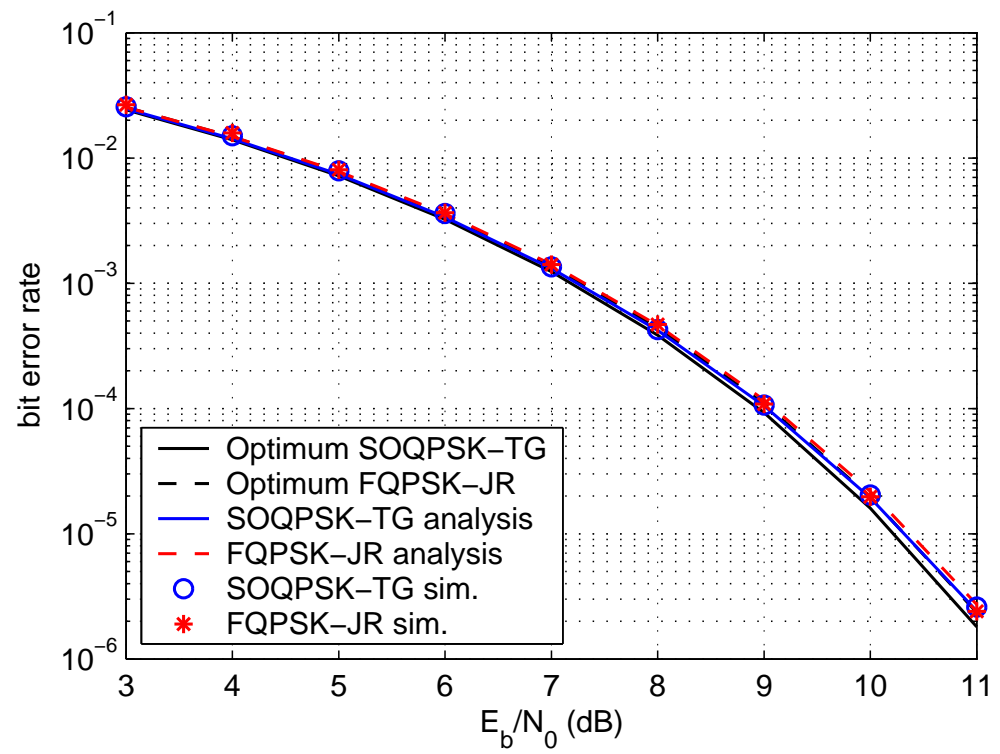

Fig. 4. Probability of bit error versus $E_{b} / N_{0}$ for the common XTCQM detector for both SOQPSK-TG and FQPSK-JR. For this detector $\Delta_{\mathrm{S}}=0.14 \mathrm{~dB}$ and $\Delta_{\mathrm{F}}=0.05 \mathrm{~dB}$.

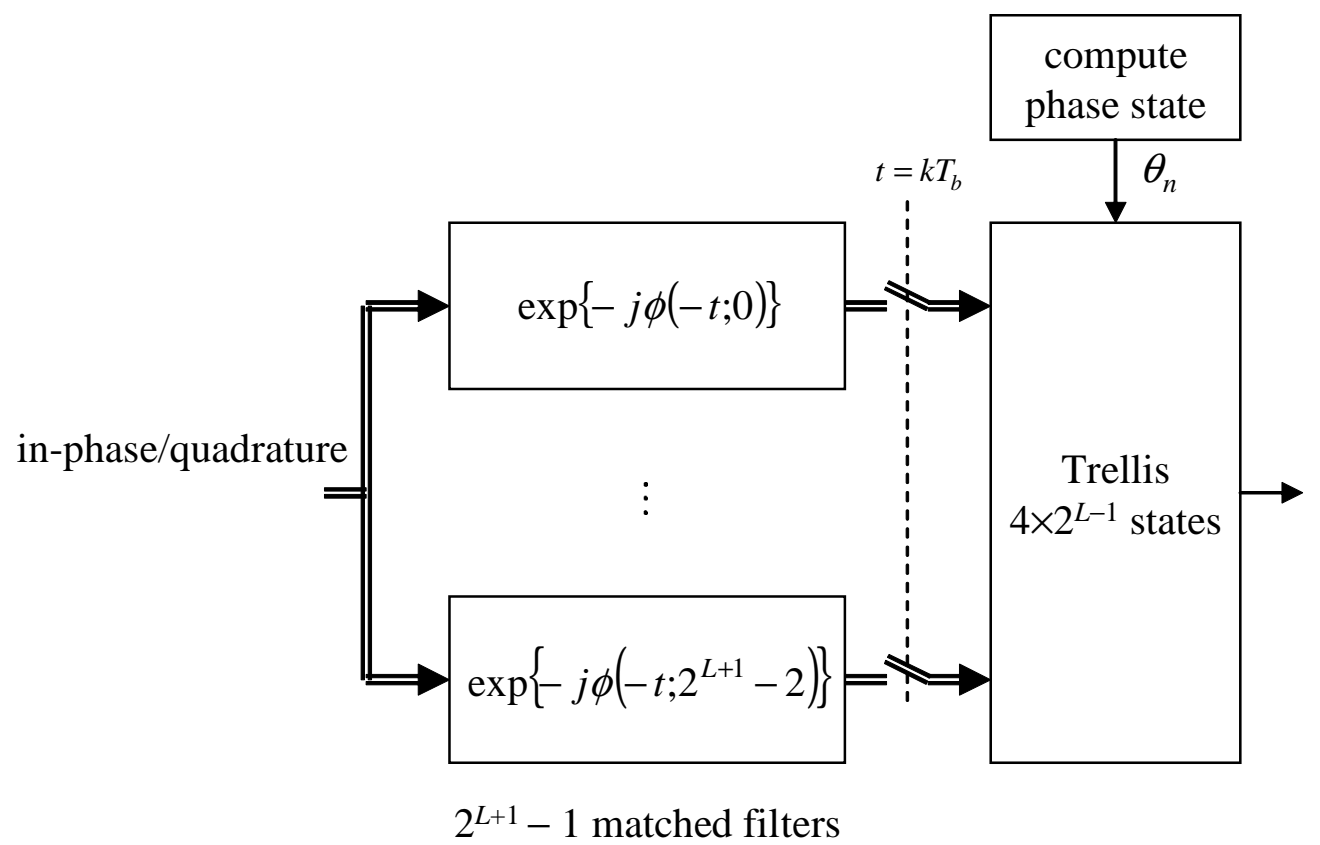

Fig. 5. The maximum likelihood CPM detector for a length- $L$ frequency pulse and ternary symbols constrained by (5). Each filter is a complex-valued filter of length $T_{b}$. The indexes in the filter impulse responses are the decimal equivalents of the binary symbol patterns that define the waveforms. 


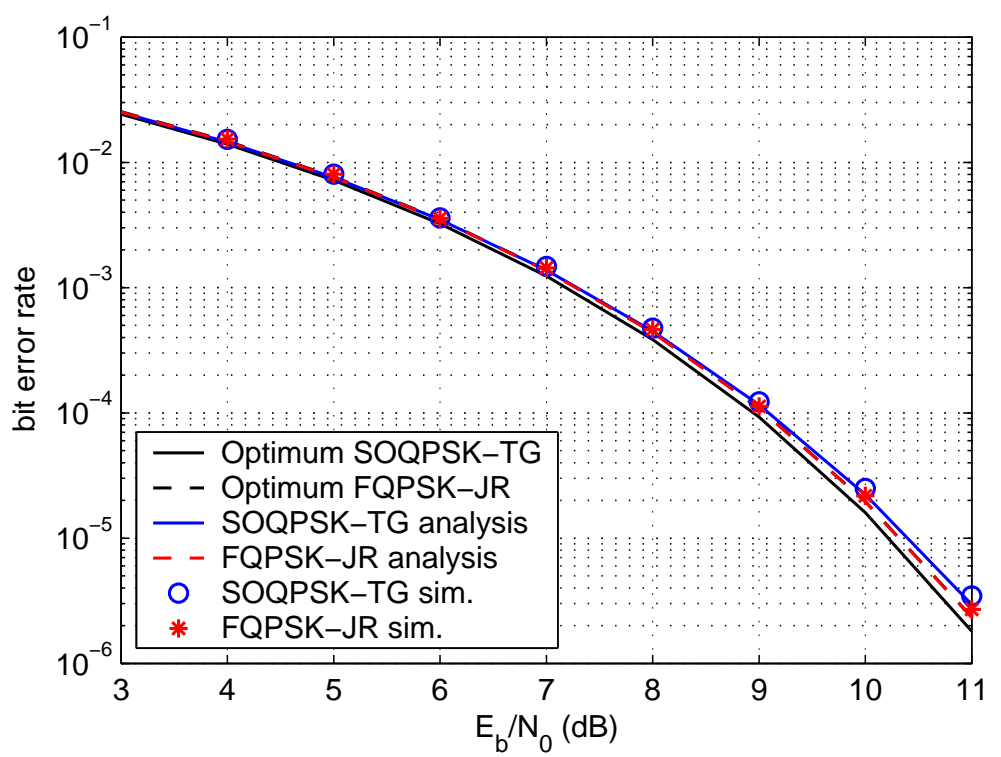

Fig. 6. Probability of bit error for SOQPSK-TG and FQPSK-JR for the common CPM detector (based on the FQPSK-JR frequency pulse) along with the theoretical curves for each modulation. Also shown are simulation results for this detector with both modulations. The common CPM detector has $\Delta_{\mathrm{S}}=0.21 \mathrm{~dB}$ and $\Delta_{\mathrm{F}}=0.01$.

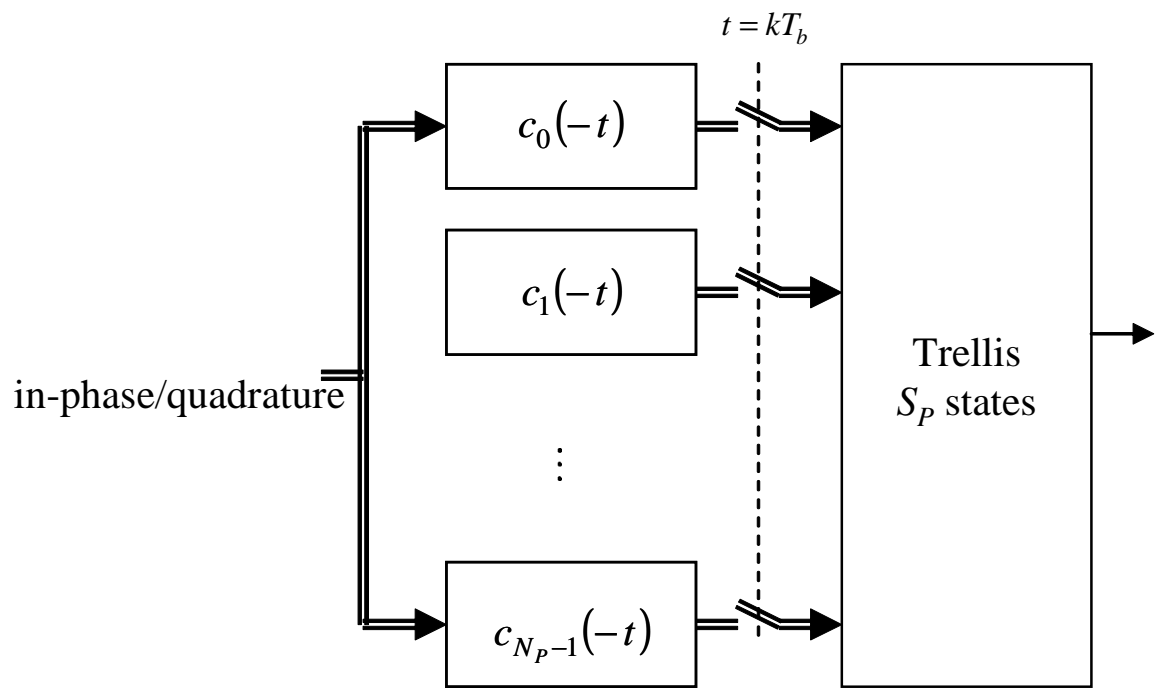

Fig. 7. The maximum likelihood detector for CPM based on the equivalent PAM representation. Each filter is a real-valued filter with varying lengths as described in the text. 


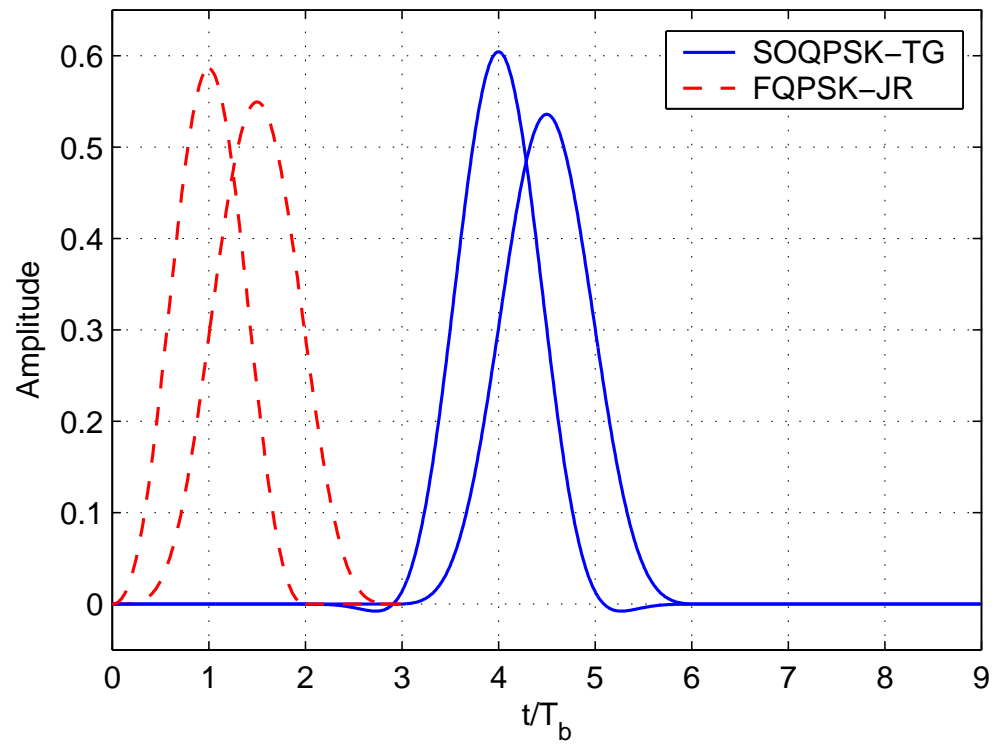

Fig. 8. Principal PAM pulses for SOQPSK-TG and FQPSK-JR.

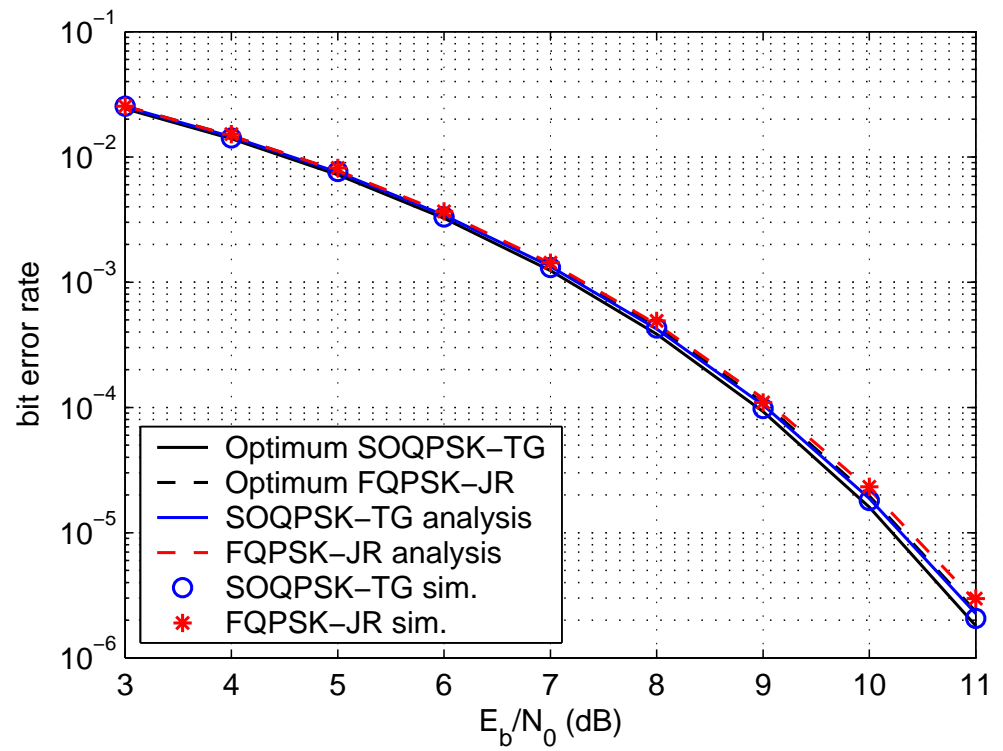

Fig. 9. Probability of bit error for the common PAM detector based on the SOQPSK-TG PAM pulses. Also shown are simulation results for both SOQPSK-TG and FQPSK-JR detected with this common PAM detector. The common PAM detector has $\Delta_{\mathrm{S}}=0.11 \mathrm{~dB}$ and $\Delta_{\mathrm{F}}=0.09$ $\mathrm{dB}$. 